\title{
III Zw 2: Evolution of a Radio Galaxy in a Nutshell
}

\author{
A. Brunthaler ${ }^{1}$, H. Falcke' ${ }^{1}$, G. C. Bower ${ }^{2}$, M. F. Aller ${ }^{3}$, H. D. Aller ${ }^{3}$, \\ H. Teräsranta ${ }^{4}$ and T. P. Krichbaum ${ }^{1}$ \\ ${ }^{1}$ Max-Planck-Institut für Radioastronomie, Auf dem Hügel 69, 53121 Bonn, Germany \\ brunthal@mpifr-bonn.mpg.de \\ ${ }^{2}$ RAL, University of California at Berkeley, 601 Campbell Hall, CA 94720, USA \\ ${ }^{3}$ University of Michigan, Astronomy Department, Ann Arbor, MI 48109-1090, USA \\ ${ }^{4}$ Metsähovi Radio Observatory, Metsähovintie, 02540 Kylmälä, Finland
}

Received 2002 July 30, accepted 2003 January 13

\begin{abstract}
III Zw 2 shows dramatic radio outbursts roughly every five years. Here we present the light curves and VLBA observations of the latest flare with an excellent time sampling. We have detected superluminal motion with a lower limit for the apparent expansion speed of $1.25 \pm 0.09 c$ at $43 \mathrm{GHz}$. Spectral and spatial evolution are closely linked and the evolution of III Zw 2 in a turnover frequency vs. linear size diagram is similar to the correlation for GPS and CSS sources. Before and after this rapid expansion we observe a period of virtually no expansion. However, at $15 \mathrm{GHz}$ III $\mathrm{Zw} 2$ shows a constant slow expansion $(\sim 0.6 c)$. The difference is qualitatively explained by optical depth effects in an 'inflating-balloon model', describing the evolution of radio lobes on an ultra-compact scale. The stop-and-go behaviour could be explained by a jet interacting with a molecular cloud or the molecular torus.
\end{abstract}

Keywords: galaxies: active — galaxies: individual (III Zw 2) — galaxies: jets

\section{Introduction}

In the currently favoured youth model for compact steep spectrum (CSS) and gigahertz peaked spectrum (GPS) sources, the linear size of a source is related to the age of the source. The correlation between the turnover frequency and the projected linear size (e.g. O'Dea \& Baum 1997) suggests that the turnover frequency decreases while the source ages and expands. Therefore the sources with the highest turnover frequencies represent the youngest objects. The origin of the turnover is probably caused by synchrotron self-absorption (SSA), while free-free absorption (FFA) could also be important in some objects such as NGC 1052 (Kadler et al. 2002; Kameno et al. 2001).

Some of the GPS/CSS have extended structure (e.g. 1245+676, Marecki et al. 2003). This indicates that they are in a phase of renewed activity and the extended structure is a product of a previous period of activity.

III Zw 2 (PG 0007+106, Mrk 1501, $z=0.089$ ) was initially classified as a Seyfert 1 galaxy (e.g. Arp 1968) but was later also included in the PG quasar sample (Schmidt \& Green 1983). It is a core-dominated AGN with a highly inverted synchrotron spectrum. With a spectral peak due to SSA around $43 \mathrm{GHz}$ during outburst, it could be classified as a millimeter peaked spectrum (MPS) source in analogy to GPS sources (Falcke et al. 1999). Its faint extended structure is typical of Seyfert galaxies (Unger et al. 1987). III Zw 2 is also part of a sample of radio intermediate quasars (RIQs). From their total flux RIQs appear to be part of the radio-loud distribution, their low extended flux indicates that they might instead be part of the radio-weak distribution. Falcke et al. (1996) and Miller et al. (1993) proposed that RIQs might be relativistically boosted radio-weak quasars. III Zw 2 varies by up to a factor of 30 over two years with major flares roughly every five years (Aller et al. 1985).

In 1997 III Zw 2 started a new outburst and we began to monitor this source with the VLA and VLBA. We observed the source 41 times from September 1998 to September 2001 with the VLA at 1.4, 4.8, 8.4, 15, 22 and $43 \mathrm{GHz}$ and 9 times from February 1998 to September 2000 with the VLBA at 15 and $43 \mathrm{GHz}$. VLA light curves at $4.8-22 \mathrm{GHz}$ are shown in Figure 1.

\section{Results}

The spectral peak - determined from fitting a broken power-law to the spectra - stayed constant around $33 \mathrm{GHz}$ during the slow and smooth rise in flux density, and we detected no structural change on VLBI scales at $43 \mathrm{GHz}$ during this time. The separation of model-fit components remained constant at $76 \mu$ as, corresponding to $0.11 \mathrm{pc}\left(H_{0}=75 \mathrm{~km} \mathrm{~s}^{-1} \mathrm{Mpc}^{-1} ; q_{0}=0\right)$. The statistical errors were estimated by dividing the original beam size by the post-model fit signal-to-noise ratio. The typical beam sizes were $0.3 \times 0.15$ mas at $43 \mathrm{GHz}$ and $0.8 \times 0.35$ mas at $15 \mathrm{GHz}$. In December 1998, the flux density started to drop rapidly. At the same time, the turnover frequency dropped quickly from $\sim 33$ to $\sim 10 \mathrm{GHz}$ in the space of a few months (see Fig. 2). Applying a simple equipartition jet model with an $R \propto v_{\mathrm{SSA}}^{-1}$ dependence, we predicted a rapid expansion during this time.

Indeed the fifth epoch of VLBA observations showed a dramatic structural change on milliarcsecond scales. The component separation increased to $245 \mu$ as or $0.36 \mathrm{pc}$. This yields an apparent expansion speed of $\sim 1.25 c$ 
Flux density [Jy]

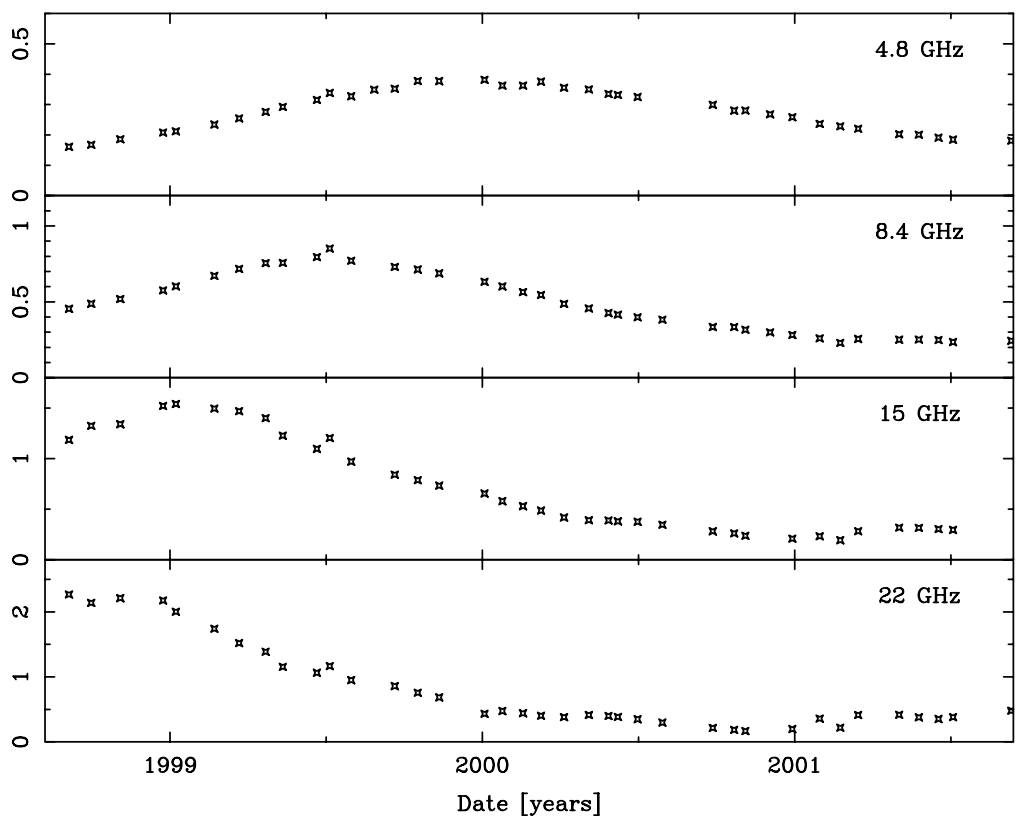

Figure 1 Light curves of III Zw 2 from 4.8 to $22 \mathrm{GHz}$ from the VLA monitoring.

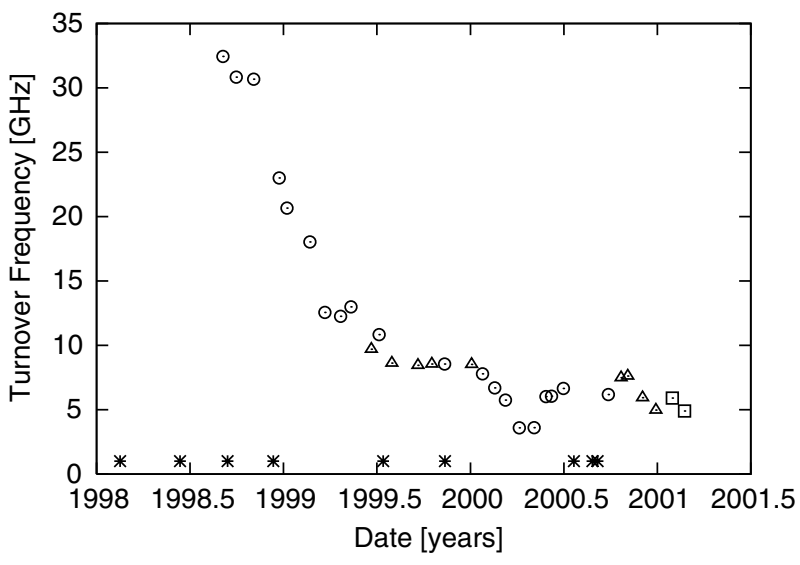

Figure 2 Evolution of the turnover frequency. The circles mark epochs with six observed frequencies $(1.4-43 \mathrm{GHz})$ and the triangles epochs with five observed frequencies $(1.4-22 \mathrm{GHz})$. In the last two epochs only four frequencies $(1.4-15 \mathrm{GHz})$ were used because of a new outburst at high frequencies. The asterisks mark the epochs of our VLBA observations.

(Brunthaler et al. 2000). After this phase of superluminal expansion and rapid spectral evolution, the expansion stopped and the spectral evolution slowed. In the last three epochs, the source was too weak at $43 \mathrm{GHz}$ to be detected.

At $15 \mathrm{GHz}$ the picture is completely different. III $\mathrm{Zw} 2$ shows a slow but constant expansion $(\sim 0.6 c)$ during the nine epochs of our monitoring program (Figure 3 ). This apparent contradiction can be explained by optical depth effects in an 'inflating-balloon model'.

\section{3 'Inflating-Balloon Model'}

In this model, the initial phase of the flux density rise can be explained by a relativistic jet interacting with the
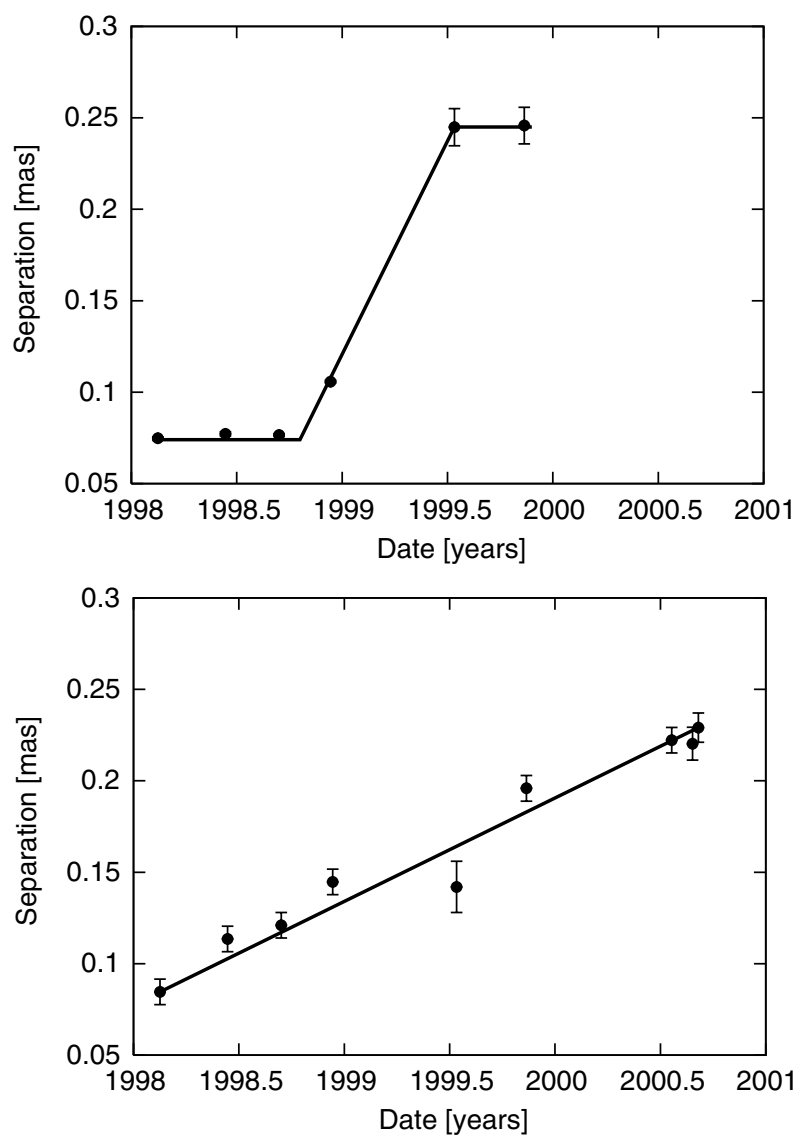

Figure 3 Component separation at 43 (upper) and 15 (lower) GHz. III Zw 2 shows no expansion during the first three epochs at $43 \mathrm{GHz}$, followed by a period of rapid expansion with an apparent expansion speed of $1.25 c$ before the jet stops again. The errorbars of the first four epochs are smaller than the plotting symbol. At $15 \mathrm{GHz}$, the source shows a constant expansion with a speed of $0.6 c$. 


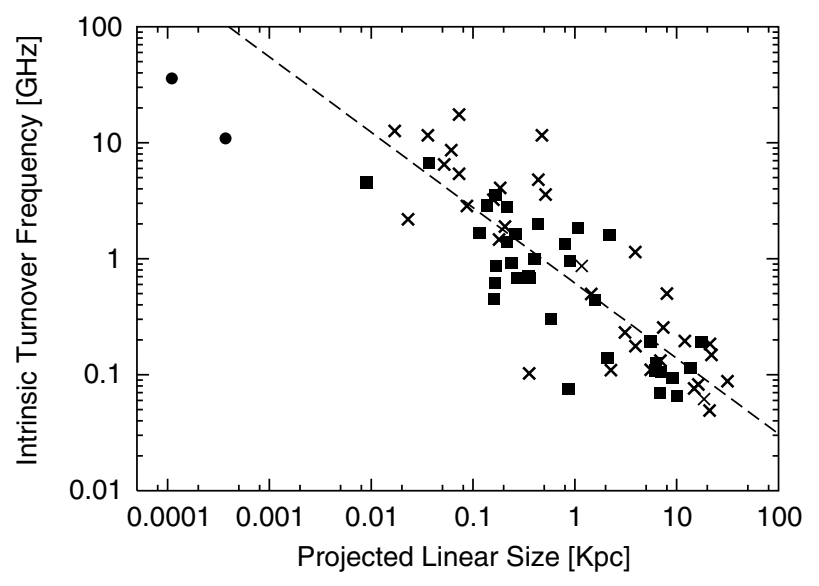

Figure 4 Intrinsic turnover frequency vs. linear size for GPS and CSS sources. The quasars are represented by crosses, and the galaxies by solid squares. Adapted from O'Dea \& Baum (1997). The two circles mark the values for III $\mathrm{Zw} 2$ before and after the expansion at $43 \mathrm{GHz}$.

interstellar medium or a torus that creates a shock. A relativistic shock was proposed earlier by Falcke et al. (1999) due to synchrotron cooling times of 14-50 days which are much shorter than the duration of the outburst. The ultracompact hotspots are pumped and powered by the jet and are responsible for the flux-density increase. The jet is frustrated and the hotspots are stationary. The post-shock material expands with the maximum sound speed of a magnetised relativistic plasma of $c_{s} \approx 0.6 c$.

Since the source is optically thick at $15 \mathrm{GHz}$, one necessarily observes the outside of the source, i.e. the post-shock material expanding at sound speed. At $43 \mathrm{GHz}$, the source is optically thin, and one can look into the source and see the stationary hotspots. This model is supported by the fact that the separation is larger at $15 \mathrm{GHz}$ (post-shock material) than at $43 \mathrm{GHz}$ (ultra-compact hotspots).

The rapid expansion at $43 \mathrm{GHz}$ thereafter marks the second phase where the jet breaks free and starts to propagate relativistically into a lower-density medium. This expansion is accompanied by the sharp drop of the turnover frequency. In the third phase, the jet is stopped again while the spectral evolution slowed down.

In Figure 4 we plot linear size vs. turnover frequency for GPS and CSS sources. We include III Zw 2 before $\left(\nu_{\mathrm{SSA}} \approx 33 \mathrm{GHz}\right.$; size $\left.\approx 0.11 \mathrm{pc}\right)$ and after $\left(\nu_{\mathrm{SSA}} \approx\right.$ $10 \mathrm{GHz}$; size $\approx 0.36 \mathrm{pc}$ ) the expansion; note that these two points lie at the lower end of the scatter of the linear correlation for GPS/CSS sources. This could be explained by projection effects. Since III Zw 2 is a Seyfert 1 galaxy with superluminal motion, the jet is probably close to the line-of-sight. Hence the true size would be underestimated, and the points in the plot move to the right. However, the evolution of III Zw 2 during the expansion is almost parallel to the correlation. This implies that the same physical processes, i.e. SSA, are involved in III Zw 2 and in GPS/CSS sources.

\section{Conclusion}

The unique and simple structure of such outbursts on a timescale of less than five years makes III Zw 2 an ideal source to study radio-jet evolution that is relevant also to radio galaxies, especially those that appear as CSOs and GPSs.

III Zw 2 remains an extremely unusual object. With the extended structure, III Zw 2 is clearly not a young source but shows intermittent activity with outbursts roughly every five years. The apparent superluminal motion confirms earlier predictions for this source, based on the argument that RIQs could be relativistically boosted jets in radio-weak quasars and Seyfert galaxies. The good agreement between structural and spectral evolution demonstrates that we are dealing with real physical expansion and not only a phase velocity.

\section{References}

Aller, H. D., Aller, M. F., Latimer, G. E., \& Hodge, R. E. 1985, ApJS, 59,513

Arp, H. 1968, ApJ, 152, 1101

Brunthaler, A., Falcke, A., Bower, G. C., Aller, M. F., Aller, H. D., Teräsranta, H., Lobanov, A. P., Krichbaum, T. P., \& Patnaik, A. R. 2000, A\&A, 357, L45

Falcke, H., Bower, G. C., Lobanov, A. P., Krichbaum, T. P., Patnaik, A. R., Aller, M. F., Aller, H. D., Teräsranta, H., Wright, M. C. H., \& Sandell, G. 1999, ApJ, 514, L17

Falcke, H., Patnaik, A. R., \& Sherwood, W. 1996, ApJ, 473, L13

Kadler, M., Ros, E., Kerp, E., Lobanov, A. P., Falcke, H., \& Zensus, J. A. 2002, in Proceedings of the 6th European VLBI Network Symposium, Ros, E., Porcas, R. W., Lobanov, A., Zensus, J. A. (eds.), (Bonn, Germany), 167-170

Kameno, S., Sawada-Satoh, S., Inoue, M., Shen, Z., \& Wajima, K. 2001 PASJ, 53, 169

Marecki, A., Barthel, P. D., Polatidis, A., \& Owsianik, I. 2003, PASA, 20, 16

Miller, P., Rawlings, S., \& Saunders, R. 1993, MNRAS, 263, 425

O’Dea, C. P. \& Baum, S. A. 1997, AJ, 113, 148

Schmidt, M. \& Green, R. F. 1983, ApJ, 269, 352

Unger, S. W., Lawrence, A., Wilson, A. S., Elvis, M., \& Wright, A. E. 1987, MNRAS, 228, 521 